\title{
BANK LIQUIDITY AND FINANCIAL PERFORMANCE: EVIDENCE FROM MOROCCAN BANKING INDUSTRY
}

\author{
El Mehdi FERROUHI \\ Faculty of Law and Economics, Mohammed V Agdal University, Avenue des Nations-Unies, \\ B.P. 721 Agdal, Rabat, Morocco \\ E-mail: elmehdiferrouhi@gmail.com
}

Received 05 March 2014; accepted 16 October 2014

\begin{abstract}
This paper aims to analyze the relationship between liquidity risk and financial performance of Moroccan banks and to define the determinants of bank's performance in Morocco during the period 2001-2012. We first evaluate Moroccan banks' liquidity positions through different liquidity and performance ratios then we apply a panel date regression to identify determinants of Moroccan banks performance. We use 4 bank's performance ratios, 6 liquidity ratios and we analyze 5 specific determinants and 5 macroeconomic determinants of bank performance.

Results show that Moroccan bank's performance is mainly determined by 7 determinants: liquidity ratio, size of banks, logarithm of the total assets squared, external funding to total liabilities, share of own bank's capital of the bank's total assets, foreign direct investments, unemployment rate and the realization of the financial crisis variable. Banks' performance depends positively on size of banks, on foreign direct investments and on the realization of the financial crisis and negatively on external funding to total liabilities, on share of own bank's capital of the bank's total assets and on unemployment rate while the dependence between bank performance and liquidity ratios and bank performance and logarithm of the total assets squared depend on the model used.
\end{abstract}

Keywords: liquidity, risk, financial performance, Morocco, determinants, size of bank, financial crisis.

JEL Classification: G17, G21, G32.

\section{Introduction}

The financial turmoil of 2007 revealed the importance of the sound liquidity risk management. Thus, the crisis that was caused by a credit crisis was transformed into a liquidity crisis. The financial crisis of 2007 , also called subprime crisis begin in the first half of 2007 with the crash of the credit quality of US subprime residential mortgages. Indeed, the decline in housing prices in the United States led to an increase in delinquencies in mortgage lending that triggered a liquidity crisis in 2007 . However, the financial crisis was not only limited to bank bankruptcies, quasi-bankruptcies, nationalizations and a decline of financial performance of large financial institutions. The financial crisis also caused a deterioration of international stock markets, a drying of liquidity in interbank markets and spilled over into a sovereign debt crisis in several European countries in early 2010 (Greece, Portugal, Ireland, Italy and Spain) (Moro 2013). Economists and policymakers who was concentrated on causes and consequences of global excess liquidity before the crisis, focused on liquidity of financial institutions, mainly banks after 2007 (Geršl, Komárková 2009).

Considered as the most severe financial crisis since the Great Depression (Brunnermeier 2009), the global financial crisis has demonstrated the importance of establishing a level of liquidity sufficient to cope with adverse conditions. These tensions in the financial markets have highlighted serious flaws in the methods of management of liquidity risk of individual banks. Liquidity is defined as the ability of a bank to fund increases in assets and meet obligations as they come due, without incurring unacceptable losses

Copyright (c) 2014 The Authors. Published by VGTU Press.

This is an open-access article distributed under the terms of the Creative Commons Attribution-NonCommercial 4.0 (CC BY-NC 4.0) license, which permits unrestricted use, distribution, and reproduction in any medium, provided the original author and source are credited. The material cannot be used for commercial purposes.

To link to this article: http://dx.doi.org/10.3846/btp.2014.443 
(Basel Committee on Banking Supervision 2008). Thus, the Third Basel Accord has reviewed the banking practices in risk management due to the subprime crisis, in order to strengthen the financial system. These agreements have given rise to two main ratios: "Liquidity Coverage Ratio (LCR) which aims to ensure that a bank maintains an adequate level of unencumbered, high-quality liquid assets that can be converted into cash to meet its liquidity needs for a 30 calendar day time horizon under a significantly severe liquidity stress scenario specified by supervisors, and Net Stable Funding Ratio (NSFR) which aims to ensure that long term assets are funded with at least a minimum amount of stable liabilities in relation to their liquidity risk profiles" (Basel Committee on Banking Supervision 2010).

The first studies on liquidity risk were mainly focused on bank runs and financial crisis (Diamond, Dybvig 1983). Researchers and practitioners were then interested in the relationship between the risk of liquidity and bank performance. According to the European Central Bank, bank's performance is the capacity to generate sustainable profitability which is essential for banks to maintain ongoing activity and for its investors to obtain fair returns; and crucial for supervisors, as it guarantees more resilient solvency ratios, even in the context of a riskier business environment (European Central Bank 2010).

Most of the empirical papers on the relationship between banks' performance and banks' liquidity examine European and Asian banks. Thus, in Europe, Molyneux and Thornton (1992) examine the determinants of bank performance of eighteen European countries between 1986 and 1989. Results show that the ratio of liquid assets to total assets is negatively related to return on assets ROA. Kosmidou et al. (2005) analyze the UK commercial banking industry over the period 1995-2002 and investigate the impact of bank's characteristics, macroeconomic conditions and financial market structure on bank's net interest margin and return on average assets ROAA. Results show that the ratio of liquid assets to customer and short term funding is positively related to return on average assets ROAA and negatively related to net interest margins NIM. Athanasoglou et al. (2006) analyze an unbalanced panel dataset of South Eastern European credit institutions over the period 1998-2002 and found that liquidity risk, measured by the ratio of loans on total assets has no effect on return on assets ROA and return on equity ROE. Pasiouras and Kosmidou (2007) study the effects of bank's specific characteristics and banking environment on the profitability of commercial domestic and foreign banks operating in the $15 \mathrm{EU}$ countries over the period 1995-2001. Results show that liquidity risk measured by the ratio of net loans to customer and short term funding is positively related to domestic banks' performance and negatively related to foreign banks' performance both measured by return on average assets (ROAA). In his paper, Kosmidou
(2008) examines the determinants of performance of 23 Greek banks during the period of EU financial integration (1990-2002). Results show that liquidity risk measured by the ratio of net loans to customer and short term funding is negatively related to performance measured by return on average assets (ROAA).

In Asia, Chen et al. (2001) analyze the banking industry in Taiwan from 1993 to 1999 to identify determinants of net interest margins in Taiwan banking industry. Results show that the ratio of liquid assets to deposits is negatively related to net interest margins NIM. Ariffin (2012) analyze the relationship between liquidity risks and Islamic banks financial performance in Malaysia over the period 2006-2008. Measuring liquidity risk by the ratio of total assets over liabilities, the author found that, in time of crisis, liquidity risk and return on assets ROA and return on equity ROE tend to behave in an opposite way and that liquidity risk may lower ROA and ROE. Naceur and Kandil (2009) analyze a sample of 28 banks over the period 1989-2004. They study the effects of capital regulations on the performance and stability of banks in Egypt. The authors found that "liquidity, measured by the ratio of net loans to customer and short term funding, is statistically significant and positively related to the profitability of domestic banks and banks' liquidity does not determine returns on assets or equity (ROA or ROE) significantly".

Other studies analyze banks from different countries. Thus, Demirgüç-Kunt and Huizinga (1999) study the determinants of bank's interest margins in 80 countries (OECD countries, developing countries and economies in transition). Results obtained show that liquidity risk measured by the ratio of loans to total assets is negatively related to return on assets ROA and positively related to net interest margins NIM. Bourke (1989) studies the internal and external determinants of profitability of twelve European, North American and Australian banks. Results show that the liquidity ratio measures by liquid assets to total assets is positively related to return on assets (ROA). Barth et al. (2003) examine the relationship between the structure, scope, and independence of bank supervision and bank profitability in 2300 banks from 55 countries. The liquidity risk measured by the ratio of liquid assets to total assets is negatively related to return on assets ROA. DemirgüçKunt et al. (2003) examine the impact of bank regulations, concentration, inflation, and national institutions on bank net interest margins NIM using data from over 1,400 banks across 72 countries. Results obtained show that liquidity risk measured by the ratio of liquid assets to total assets is negatively related to net interest margins NIM. Chen et al. (2009) investigate the determinants of bank performance in terms of the perspective of the bank liquidity risk. The authors use an unbalanced panel dataset of 12 advanced economies commercial banks (Australia, Canada, 
France, Germany, Italy, Japan, Luxembourg, Netherlands, Switzerland, Taiwan, United Kingdom and United States) over the period 1994-2006 to estimate the causes of liquidity risk model. Results obtained show that liquidity risk is the endogenous determinant of bank performance measured by return on assets average, return on equity average and net interest margins and that liquidity risk is negatively related to return on assets average ROAA and return on equity average ROEA and positively related to net interest margins NIM.

The aim of the present paper is to analyze the relationship between liquidity risk and financial performance of Moroccan banks and to define the determinants of bank's performance in Morocco. For this purpose, we use a panel data regression of Moroccan banks during the period 2001-2012. We use 4 bank's performance ratios (return on assets, return on equity, return on average assets and net interest margins), 6 liquidity ratios (liquid assets to total assets, liquid assets to short term liabilities, liquid assets to deposits, loans to total assets, loans to deposits and short term liabilities and the ratio of financing gap to total assets) and we also analyze 5 specific determinants (logarithm of the total assets of the bank to measure the size of banks, logarithm of the total assets squared to capture the nonlinear relationship, share of own bank's capital of the bank's total assets, external funding to total liabilities and equity to total assets) and 5 macroeconomic determinants of bank performance (unemployment rate, inflation rate, growth rate of gross domestic product; foreign direct investment and a variable that we simulated for detecting the realization of the financial crisis).

The paper is organized as follows. In section 1 we present the model and data used in the present paper. Results obtained are presented in section 2. Finally, the last section offers conclusions.

\section{Methodology}

The purpose of this paper is to analyze the relationship between liquidity risk and financial performance of Moroccan banks and to define the determinants of bank's performance in Morocco. To do this, we first evaluate Moroccan bank's liquidity positions and bank's financial performance through different liquidity ratios. We then identify determinants of Moroccan bank's performance using a panel data regression and analyze the relationship between liquidity risk and financial performance of Moroccan banks.

Vento and La Ganga (2009) defined three methods to measure liquidity risk: the stock approach which looks at liquidity as a stock, the cash-flows based approach which aims to safeguard the bank's ability to meet its payment obligations and calculating and limiting the liquidity maturity transformation risk and the hybrid approaches which combines elements of the stock approach and of the cashflows based approaches.

Basel Committee on Banking Supervision proposed maturity laddering method, that allows comparing cash inflows and outflows both on a day-to-day basis and over a series of specified time periods as a measure of liquidity risk (Basel Committee on Banking Supervision 2000) while some authors proposed the use of peer group ratio comparisons, liquidity index, financing gap (Saunders, Cornett 2006) and balance sheet liquidity analysis, cash capital position and maturity mismatch approach (Matz, Neu 2006) as measures of liquidity exposure. However, Poorman and Blake (2005) indicate that the use of just ratios to measure liquidity was insufficient justifying by the fact that banks with positive liquidity ratio can go bankrupt (Southeast Bank of Miami in 1991). The authors propose financing gap measures to assess bank liquidity risk.

As various authors provide the use of the stock approach (Yeager, Seitz 1989; Hemple, Simonson 2008; Fielding, Shortland 2005; Lucchetta 2007; Moore 2010) which is the more popular both in the academic literature and in practice, we use in this paper following ratios:

- $L_{1}=\frac{\text { Liquid assets }}{\text { Total assets }} \times 100$ which measures the ability of a bank to absorb liquidity shocks. A high ratio means a high ability to absorb shocks;

- $L_{2}=\frac{\text { Liquid assets }}{\text { SHort term liabilities }} \times 100$ measures the ability of a bank to cope a high demand of short term liquidity. A high ratio means that the bank is liquid at short-term;

- $L_{3}=\frac{\text { Liquid assets }}{\text { Deposits }} \times 100$ used to measure bank's liquidity in the case that the bank cannot borrow from other banks. A high ratio means that the bank is able to cope long term liquidity risk;

- $L_{4}=\frac{\text { Loans }}{\text { Total assets }} \times 100$ which measures the share of loans in total assets. It shows the percentage of the bank's assets related to illiquid loans. When this ratio is high, it means that the bank is less liquid;

$-L_{5}=\frac{\text { Loans }}{\text { Deposits }+ \text { SHort term liabilities }} \times 100$ indicates the relationship of illiquid assets and liquid liabilities. When this ratio is high, it means that the bank is less liquid;

- $L_{6}=\frac{\text { Bank's loans }- \text { customer deposits }}{\text { Total assets }} \times 100 \quad$ which measures liquidity risk exposure. Defined as the difference between a bank's loans and customer deposits, financing gap is divided by total assets to standardize and get the ratio of financing gap to total assets (FGAPR). 
In the other hand, as defined above, bank's performance is the capacity to generate sustainable profitability. The European Central Bank defines 3 traditional measures of performance: return on assets ROA, return on equity ROE and net interest margins NIM. As presented in the literature review, authors use different banks' performance ratios especially ROA, ROE, ROAA and NIM. In the present paper, we use all this different ratios to measure Moroccan bank's performance:

- ROA $=\frac{\text { Net income }}{\text { Total assets }} \times 100$ measures bank's profitability relative to its assets and thus the bank's overall performance;

- $\mathrm{ROE}=\frac{\text { Net income }}{\text { Shareholder equity }} \times 100$ measures a corporation's profitability by revealing how much profit a company generates with the money shareholders have invested;

- ROAA $=\frac{\text { Net income }}{\text { Average total assets }} \times 100$ reflects the ability of a bank's management to generate profits from the bank's assets;

- NIM $=\frac{\text { Total interest income }- \text { Total interest expense }}{\text { Total earning assets }} \times$ 100 measures the gap between what the bank pays savers and what the bank receives from borrowers.

To define Moroccan performance, our methodology consists first on the calculation of different ratios presented above for Moroccan banks during the period 2001-2012. We use Augmented Dickey Fuller (Dickey, Fuller 1979) test to test the stationary. The null hypothesis of nonstationary is rejected at the $5 \%$ level. We then use a panel data regression with fixed effects. Thus, we estimate for each of the previously defined performance ratios the following equation:

$$
P_{i t}=c+\lambda \cdot L_{i t}+\sum_{i}^{N} \beta_{m} \cdot X_{i t}+\delta_{i}+\varepsilon_{i t},
$$

with: $L_{i t}$ one of different performance ratios for bank i at time $t, c$ a constant; $L_{i t}$ liquidity risk ratio for bank $i$ at time $\mathrm{t} ; X_{i t}$ vector of explanatory variables for bank $\mathrm{i}$ at time $\mathrm{t} ; \beta$ coefficient which represents the slope of variables; $\delta_{i}$ fixed effects on the bank $i$ and $\varepsilon_{\text {it }}$ the error term.

Extending equation (1) to reflect all the variables, we obtain the following model:

$$
\begin{aligned}
& P_{i t}=c+\lambda L_{i t}+\beta_{1} L A G A_{i t}+\beta_{2} L A G A_{i t}{ }^{2}+\beta_{3} C T A_{i t}+ \\
& \beta_{4} E F L_{i t}+\beta_{5} E T A_{i t}+\beta_{6} \cdot U N E_{t}+\beta_{7} I N F_{t}+\beta_{8} G D P_{t}+ \\
& \beta_{9} F D I_{t}+\beta_{10} F I C_{t}+\delta_{i}+\varepsilon_{i t} .
\end{aligned}
$$

We select the most appropriate explanatory variables according to previous studies to define the determinants of
Moroccan banks performance. The explanatory variables that we use in this study are: logarithm of the total assets of the bank LAGA to measure the size of banks; logarithm of the total assets squared LAGA ${ }^{2}$ to capture the non-linear relationship; share of own bank's capital of the bank's total assets CTA; external funding to total liabilities EFL; equity to total assets ETA; unemployment rate UNE; inflation rate INF; growth rate of gross domestic product GDP; foreign direct investment FDI and a variable that we simulated for detecting the realization of the financial crisis FIC. The value of this variable is 1 for the years 2008, 2009 and 2010 and 0 for the other years. Specific variables statistics are obtained from banks' annual reports and banks' annual financial statements (LAGA, LAGA ${ }^{2}$, CTA, EFL, ETA), while macroeconomic variables statistics (UNE, INF, GDP, FDI and FIC) are obtained from the databases of the World Bank, the International Monetary Fund and the Moroccan High Commission for Planning.

The data used in this paper are obtained from annual reports and annual financial statements of the commercial Moroccan banks for the period 2001-2012 and from databases of the World Bank, the International Monetary Fund and the Moroccan High Commission for Planning. Our study concerns Moroccan commercial banks, thus we have listed the various existing banks in Morocco in the last decade during a minimum of 7 years to capture the effects of the financial crisis. We then selected banks that have existed throughout the study period and whose financial statements are available. We obtained 8 banks which are the largest Moroccan banks (see Table 1).

Table 1. List of commercial Moroccan banks for the period 2001-2012

\begin{tabular}{|l|c|}
\hline & Banks' foundation year \\
\hline ATTIJARIWAFA BANK (AWB) & 2003 \\
\hline $\begin{array}{l}\text { BANQUE CENTRALE } \\
\text { POPULAIRE (BCP) }\end{array}$ & 1961 \\
\hline $\begin{array}{l}\text { BANQUE MAROCAINE DU } \\
\text { COMMERCE EXTERIEUR } \\
\text { (BMCE BANK) }\end{array}$ & 1959 \\
\hline $\begin{array}{l}\text { BANQUE MAROCAINE } \\
\text { POUR LE COMMERCE ET } \\
\text { LINDUSTRIE (BMCI) }\end{array}$ & 1964 \\
\hline $\begin{array}{l}\text { CREDIT AGRICOLE DU } \\
\text { MAROC (CAM) }\end{array}$ & 1997 \\
\hline CREDIT DU MAROC (CDM) & 1929 \\
\hline $\begin{array}{l}\text { CREDIT IMMOBILIER ET } \\
\text { HOTELIER (CIH) }\end{array}$ & 1920 \\
\hline $\begin{array}{l}\text { SOCIETE GENERALE } \\
\text { MAROCAINE DE BANQUES } \\
\text { (SGMB) }\end{array}$ & 1913 \\
\hline NUMBER OF BANKS & 8 \\
\hline
\end{tabular}




\section{Results}

Table 2 presents determinants of return on assets ROA measured using liquidity ratios L1 to L6. We remark that the explanatory power of these models is moderate. Results show that return on assets ROA is negatively correlated with liquidity ratios L3 (at the 1\% level) and L6 (at the 5\% level). Results also show that return on assets ROA is negatively correlated with external funding to total liabilities EFL in models using L3 and L6 liquidity ratios (at the 10\% level). However, return on assets ROA is positively correlated with logarithm of the total assets squared LAGA ${ }^{2}$ in models using L1 and L6 liquidity ratios (at the 10\% level) and with foreign direct investments FDI in model using L5 liquidity ratio.

Table 3 presents determinants of return on equity ROE. We remark that the explanatory power is moderate for model using L1 and fairly strong for models using L2 to L6 liquidity ratios. Performance ratio return on equity ROE is positively correlated with logarithm of the total assets squared LAGA ${ }^{2}$ in models using L1 (at the 1\% level) and in model using L5 (at the 5\% level) and with foreign direct investment FDI (at the $10 \%$ level). In the other side, we remark that share of own bank's capital of the bank's total assets CTA is negatively correlated with return on equity in models using L1 and L3 (at the 10\% level), L2 and L4 (at the 5\% level) and L6 (at the $1 \%$ level). Return on equity is also negatively correlated with external funding to total liabilities EFL in models using liquidity ratios L1 (at the 1\% level) and L3 (at the 10\% level), with the realization of financial crisis variable FIC in models using liquidity ratios L2, L3, L4, and L5 (at the 5\% level) and L6 (at the 10\% level) and with unemployment rate UNE in models using L1 (at the 10\% level), L2, L4 and L6 (at the 5\% level) and L5 (at the 1\% level).

Table 4 presents determinants of net interest margins NIM. We note that the explanatory power of these models is strong. Results show that net interest margins NIM is negatively correlated with logarithm of the total assets squared $\mathrm{LAGA}^{2}$ in models using L1 and L4 (at the 10\% level) and L2, L3 and L6 (at the 5\% level). We remark that share of own bank's capital of the bank's total assets CTA is negatively correlated with net interest margins in model using L4 (at the $10 \%$ level). Net interest margins NIM is positively correlated with logarithm of the total assets of the bank LAGA in models using L1 and L2 liquidity ratio (at the $10 \%$ level).

Table 5 presents determinants of return on assets average ROAA. We note that the explanatory power of these models is moderate. Results show that return on assets average ROAA is negatively correlated with external funding to total liabilities EFL in models using L3 and L6 (at the 10\% level). However, logarithm of the total assets squared LAGA2 (at the $10 \%$ level) is positively correlated with return on assets average. Return on assets average is positively correlated with liquidity ratios L1 and L6 (at the 5\% level) and L3 (at the $1 \%$ level).
From different results above, we remark that return on assets is positively correlated with logarithm of the total assets squared LAGA ${ }^{2}$ and with foreign direct investment FDI and negatively correlated with liquidity ratios and with external funding to total liabilities EFL. We remark also that return on equity is positively correlated with foreign direct investment FDI and with logarithm of the total assets squared LAGA ${ }^{2}$. Return on equity is negatively correlated with external funding to total liabilities EFL, with share of own bank's capital of the bank's total assets CTA, with unemployment rate and with the realization of financial crisis variable FIC. However, we remark that logarithm of the total assets squared is positively correlated with return on equity in model using L1 as liquidity ratio and negatively correlated in model using L5 as liquidity ratio.

Net interest margin is negatively correlated with logarithm of the total assets squared LAGA ${ }^{2}$ and share of own bank's capital of the bank's total assets CTA. Net interest margin is positively correlated with to logarithm of the total assets of the bank LAGA, while return on assets average is positively correlated with liquidity ratios and logarithm of the total assets squared and negatively correlated with external funding to total liabilities.

Thus, we conclude that Moroccan bank's performance is mainly determined by 7 determinants: liquidity ratio, size of banks, logarithm of the total assets squared, external funding to total liabilities, share of own bank's capital of the bank's total assets, foreign direct investments, unemployment rate and the realization of the financial crisis variable. Banks' performance depends positively on size of banks, on foreign direct investments and on the realization of the financial crisis and negatively on external funding to total liabilities, on share of own bank's capital of the bank's total assets and on unemployment rate while the dependence between bank performance and liquidity ratios and bank performance and logarithm of the total assets squared depend on the model used.

Indeed, since liquidity is positively correlated to the bank performance in model using return on assets average and negatively correlated in model using return on assets, we cannot say that liquid banks are more efficient than banks. Large banks and banks with a low share of own bank's capital of the bank's total assets are more efficient while banks depending on external funding are less efficient. Concerning macroeconomic determinants, bank performance decreases with the financial crisis and increases when the foreign direct investments grow. Moroccan banking industry was thus impacted by the financial crisis. Bank's performance increase when unemployment rate decreases. However, the correlation of logarithm of the total assets squared and bank's performance depends on liquidity ratio used, while equity to total assets and growth rate of gross domestic product have no impact on bank's performance. 


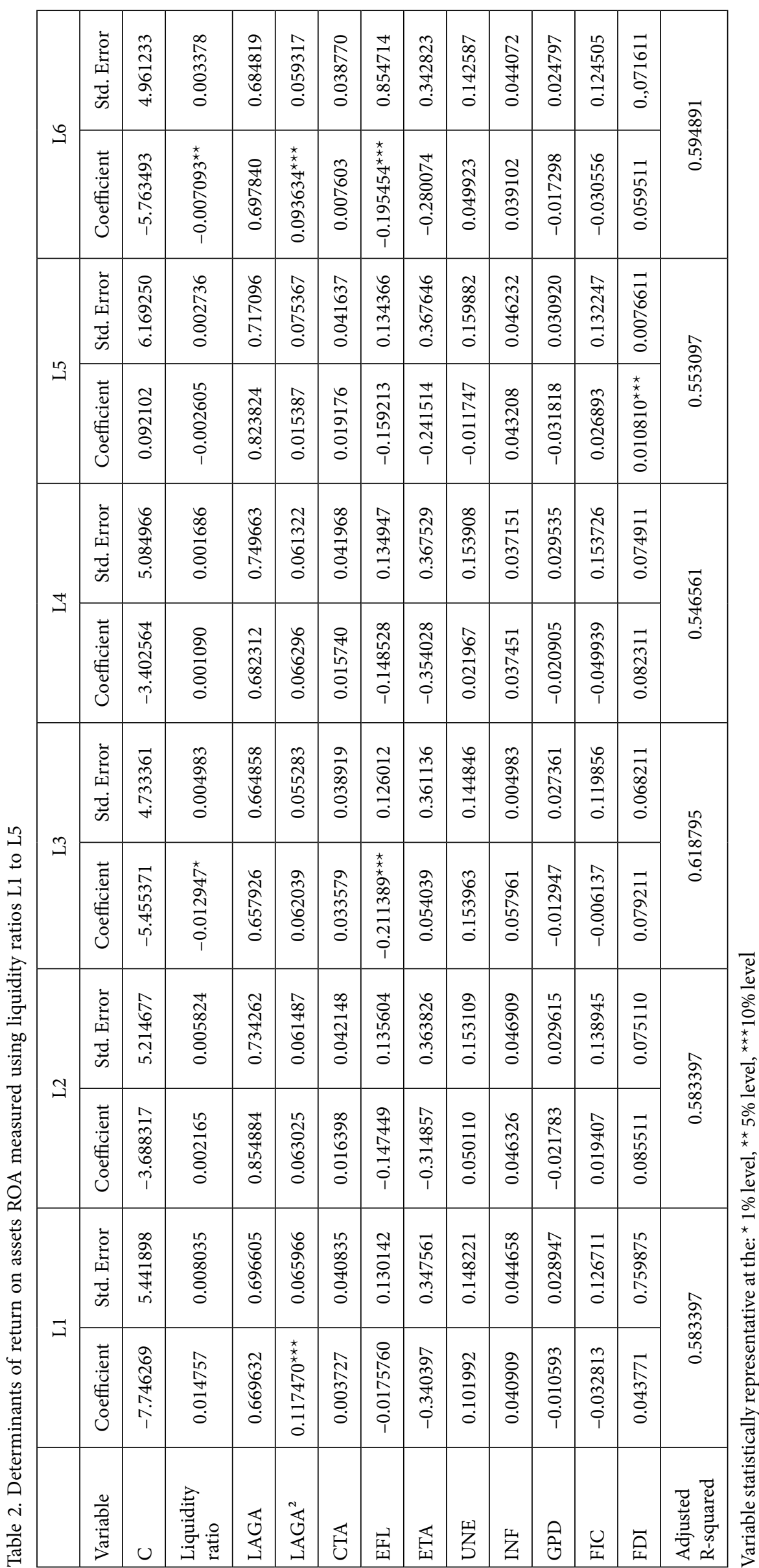




\begin{tabular}{|c|c|c|c|c|c|c|c|c|c|c|c|c|c|c|}
\hline \multirow{2}{*}{ 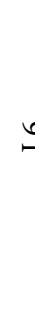 } & 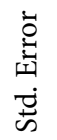 & 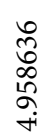 & 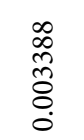 & $\begin{array}{l}\text { Oे } \\
0 \\
0 \\
0 \\
0 \\
0\end{array}$ & 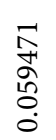 & 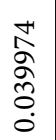 & 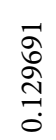 & $\underset{\substack{n \\
\multirow{2}{*}{}}}{\stackrel{n}{0}}$ & 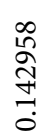 & \begin{tabular}{l}
0 \\
$\infty$ \\
\multirow{H}{*}{} \\
0 \\
0
\end{tabular} & $\begin{array}{l}\overrightarrow{\widetilde{S}} \\
\mathbf{0} \\
\stackrel{0}{0} \\
\stackrel{0}{0}\end{array}$ & 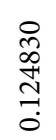 & $\begin{array}{l}\vec{\nabla} \\
\stackrel{\infty}{\Delta} \\
\stackrel{0}{0} \\
0\end{array}$ & \multirow{2}{*}{ 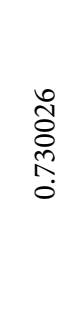 } \\
\hline & 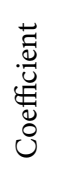 & 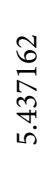 & $\begin{array}{l}8 \\
8 \\
\circ \\
0 \\
0\end{array}$ & 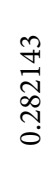 & 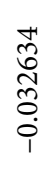 & 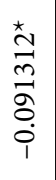 & $\begin{array}{l}\stackrel{尺}{1} \\
\infty \\
\\
0 \\
1\end{array}$ & 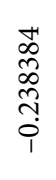 & 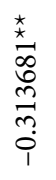 & $\begin{array}{l}\hat{n} \\
\infty \\
\overrightarrow{0} \\
0 \\
0 \\
i\end{array}$ & 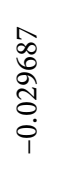 & 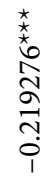 & $\begin{array}{l}\vec{\nabla} \\
\stackrel{0}{0} \\
0 \\
0\end{array}$ & \\
\hline \multirow{2}{*}{12} & 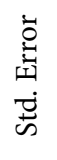 & 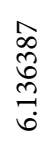 & 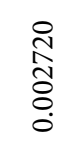 & $\begin{array}{l}\stackrel{0}{人} \\
\stackrel{1}{N} \\
\stackrel{1}{0} \\
0\end{array}$ & 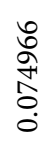 & $\begin{array}{l}\frac{n}{n} \\
\vec{H} \\
\vec{H} \\
0\end{array}$ & $\begin{array}{l}\vec{n} \\
0 \\
0 \\
0 \\
0\end{array}$ & $\begin{array}{l}\infty \\
\infty \\
0 \\
\ell \\
0 \\
0 \\
0 \\
0\end{array}$ & $\begin{array}{l}0 \\
0 \\
0 \\
0 \\
0 \\
0\end{array}$ & $\begin{array}{l}2 \\
0 \\
0 \\
4 \\
0 \\
0 \\
0\end{array}$ & $\begin{array}{l}\stackrel{0}{1} \\
0 \\
0 \\
0 \\
0 \\
0\end{array}$ & $\begin{array}{l}\frac{a}{n} \\
\stackrel{n}{n} \\
\stackrel{0}{0}\end{array}$ & $\begin{array}{l}\overrightarrow{\widehat{J}} \\
0 \\
0 \\
0\end{array}$ & \multirow{2}{*}{$\begin{array}{l}8 \\
\stackrel{0}{0} \\
\stackrel{0}{0} \\
\stackrel{0}{0} \\
0\end{array}$} \\
\hline & 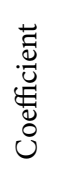 & $\begin{array}{l}\stackrel{\star}{\star} \\
\stackrel{\infty}{\infty} \\
\stackrel{+}{=} \\
\dot{m} \\
=\end{array}$ & $\begin{array}{l}N \\
\infty \\
\infty \\
\stackrel{0}{0} \\
\dot{0} \\
1\end{array}$ & $\begin{array}{l}\frac{m}{2} \\
\vec{J} \\
0\end{array}$ & 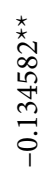 & $\begin{array}{l}\stackrel{\infty}{+} \\
\stackrel{\infty}{\infty} \\
\stackrel{0}{0} \\
\dot{\varphi} \\
1\end{array}$ & $\begin{array}{l}m \\
\underset{F}{F} \\
\stackrel{+}{+}\end{array}$ & $\begin{array}{l}8 \\
8 \\
0 \\
0 \\
0 \\
1\end{array}$ & 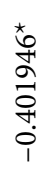 & 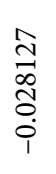 & 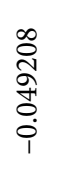 & 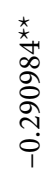 & 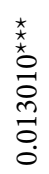 & \\
\hline \multirow{2}{*}{+} & 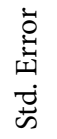 & 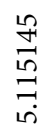 & 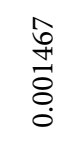 & \begin{tabular}{l}
$\sqrt[N]{\sigma}$ \\
\multirow{H}{N}{} \\
$\hat{\sigma}$
\end{tabular} & $\begin{array}{l}\stackrel{0}{0} \\
0 \\
0 \\
0 \\
0\end{array}$ & 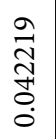 & $\begin{array}{l}n \\
\stackrel{n}{n} \\
\hat{n} \\
\stackrel{n}{0} \\
0\end{array}$ & 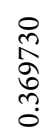 & $\begin{array}{l}0 \\
\infty \\
\infty \\
0 \\
0 \\
0 \\
0\end{array}$ & 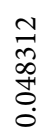 & 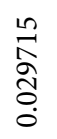 & 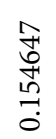 & 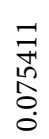 & \multirow{2}{*}{$\begin{array}{l}\text { N} \\
\hat{N} \\
\text { م⿱ } \\
0 \\
0\end{array}$} \\
\hline & 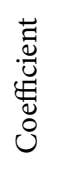 & 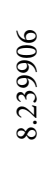 & $\begin{array}{l}\hat{o} \\
\stackrel{+}{1} \\
\dot{8} \\
0 \\
0\end{array}$ & 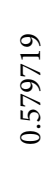 & $\begin{array}{l}\hat{\imath} \\
\tilde{n} \\
\hat{0} \\
\dot{i}\end{array}$ & 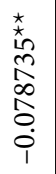 & $\begin{array}{l}\stackrel{\Omega}{2} \\
\hat{0} \\
0 \\
0 \\
1\end{array}$ & 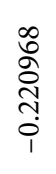 & 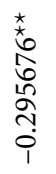 & $\begin{array}{l}\hat{0} \\
\text { A } \\
0 \\
0 \\
0 \\
i\end{array}$ & $\begin{array}{l}0 \\
0 \\
0 \\
0 \\
\delta \\
0 \\
i \\
i\end{array}$ & 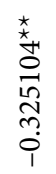 & 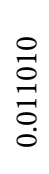 & \\
\hline \multirow{2}{*}{$\stackrel{3}{9}$} & 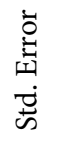 & 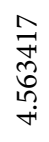 & 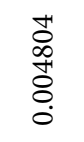 & 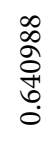 & 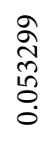 & $\begin{array}{l}\tilde{N} \\
\stackrel{n}{n} \\
\hat{N} \\
0 \\
0\end{array}$ & 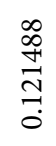 & 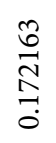 & \begin{tabular}{l}
0 \\
\multirow{1}{0}{} \\
$\stackrel{2}{0}$ \\
$\stackrel{0}{0}$
\end{tabular} & 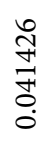 & $\begin{array}{l}\infty \\
\stackrel{\infty}{0} \\
\stackrel{0}{0} \\
o \\
0\end{array}$ & 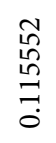 & $\begin{array}{l}= \\
- \\
0 \\
0 \\
0 \\
0 \\
0\end{array}$ & \multirow{2}{*}{ 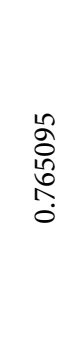 } \\
\hline & 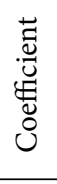 & $\begin{array}{l}\text { 응 } \\
\text { 人ิ } \\
\text { in } \\
\text { in }\end{array}$ & $\begin{array}{l}\vec{N} \\
\hat{N} \\
\vdots \\
0 \\
0\end{array}$ & $\begin{array}{l}\vec{N} \\
\stackrel{N}{N} \\
0\end{array}$ & $\begin{array}{l}n \\
2 \\
0 \\
8 \\
0 \\
0 \\
0 \\
1\end{array}$ & 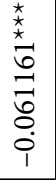 & 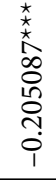 & $\begin{array}{l}\stackrel{n}{0} \\
\stackrel{\mathbb{N}}{0} \\
0\end{array}$ & 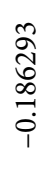 & 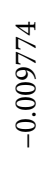 & $\begin{array}{l}m \\
\stackrel{n}{a} \\
\vec{\sigma} \\
\dot{i} \\
i\end{array}$ & 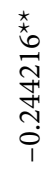 & $\begin{array}{l}\exists \\
\bar{n} \\
2 \\
0 \\
0 \\
0\end{array}$ & \\
\hline \multirow{2}{*}{$\Im$} & 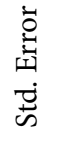 & 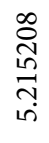 & $\begin{array}{l}\stackrel{1}{0} \\
\infty \\
0 \\
0 \\
0 \\
0\end{array}$ & 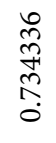 & $\begin{array}{l}\stackrel{+}{\sigma} \\
\stackrel{d}{0} \\
\dot{0}\end{array}$ & $\begin{array}{l}\tilde{N} \\
\stackrel{\sim}{\mathcal{H}} \\
0 \\
\dot{0}\end{array}$ & $\begin{array}{l}\infty \\
\overrightarrow{0} \\
\stackrel{n}{0} \\
\overrightarrow{0}\end{array}$ & $\begin{array}{l}\tilde{b} \\
\infty \\
\tilde{b} \\
\tilde{ల} \\
0\end{array}$ & $\begin{array}{l}\stackrel{\mathscr{n}}{\mathbb{N}} \\
\stackrel{\tilde{n}}{\hat{n}} \\
\stackrel{0}{0}\end{array}$ & 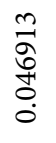 & 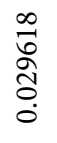 & $\begin{array}{l}\hat{n} \\
2 \\
\infty \\
\stackrel{0}{0} \\
0\end{array}$ & $\begin{array}{l}\exists \\
\exists \\
\\
\stackrel{\Xi}{0} \\
\dot{0}\end{array}$ & \multirow{2}{*}{ 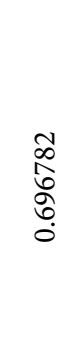 } \\
\hline & 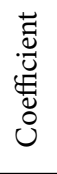 & 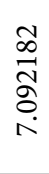 & $\begin{array}{l}\text { 号 } \\
\text { 岁 } \\
\text { Oे } \\
0\end{array}$ & $\underset{\Xi}{\stackrel{\Xi}{\Xi}}$ & $\begin{array}{l}\infty \\
\hat{N} \\
\hat{\alpha} \\
0 \\
0 \\
i\end{array}$ & 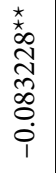 & 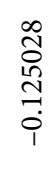 & 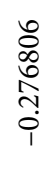 & 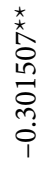 & 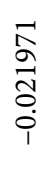 & $\begin{array}{l}\widetilde{1} \\
\infty \\
0 \\
\tilde{0} \\
0 \\
i\end{array}$ & 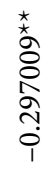 & $\begin{array}{l}\exists \\
\tilde{N} \\
\stackrel{\sigma}{0} \\
0\end{array}$ & \\
\hline & $\begin{array}{l}\overrightarrow{0} \\
\stackrel{\vec{n}}{1} \\
\vec{D} \\
\dot{\omega}\end{array}$ & 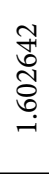 & $\begin{array}{l}\hat{\alpha} \\
\text { oे } \\
\text { ठ̣ } \\
0\end{array}$ & 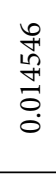 & 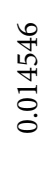 & 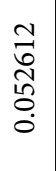 & \begin{tabular}{l}
$\stackrel{a}{0}$ \\
\multirow{J}{*}{} \\
0 \\
0
\end{tabular} & 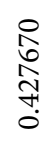 & \begin{tabular}{l}
\multirow{2}{*}{} \\
$\stackrel{0}{0}$ \\
0 \\
$\vdots$ \\
0
\end{tabular} & 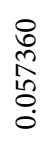 & $\begin{array}{l}\frac{1}{6} \\
0 \\
0 \\
0 \\
0\end{array}$ & $\begin{array}{l}\vec{H} \\
\infty \\
\infty \\
\\
0 \\
0\end{array}$ & 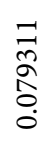 & \multirow{2}{*}{$\begin{array}{l}\text { I } \\
\text { d } \\
0 \\
0 \\
0\end{array}$} \\
\hline & 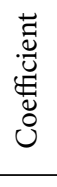 & $\begin{array}{l}\underset{f}{N} \\
\stackrel{+}{\hat{N}} \\
\stackrel{i}{i}\end{array}$ & 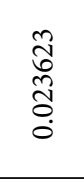 & 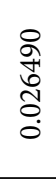 & 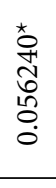 & 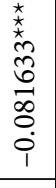 & $\begin{array}{l}\stackrel{\star}{N} \\
\widetilde{N} \\
0 \\
0 \\
0 \\
0 \\
i\end{array}$ & $\begin{array}{l}\stackrel{0}{0} \\
\stackrel{n}{n} \\
\stackrel{1}{0}\end{array}$ & 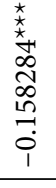 & $\begin{array}{l}+ \\
0 \\
0 \\
0 \\
0 \\
0 \\
i\end{array}$ & $\begin{array}{l}\vec{~} \\
\hat{a} \\
\stackrel{0}{0} \\
\dot{0} \\
1\end{array}$ & 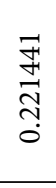 & $\begin{array}{l}\vec{I} \\
\stackrel{\Xi}{0} \\
\dot{0}\end{array}$ & \\
\hline & 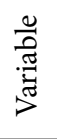 & $u$ & 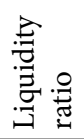 & 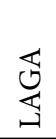 & 岕 & 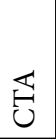 & 虹 & $\underset{|r|}{\overleftrightarrow{\mid r}}$ & 茨 & 㞱 & 今ે & $\underset{I}{U}$ & $\overrightarrow{\vec{\imath}}$ & 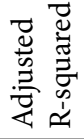 \\
\hline
\end{tabular}




\begin{tabular}{|c|c|c|c|c|c|c|c|c|c|c|c|c|c|c|}
\hline \multirow{2}{*}{$\stackrel{\ddots}{\sigma}$} & 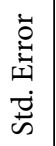 & \begin{tabular}{l}
$\hat{N}$ \\
$\exists$ \\
\multirow{H}{0}{} \\
0 \\
0
\end{tabular} & $\begin{array}{l}\text { 号 } \\
0 \\
0 \\
0 \\
0 \\
0\end{array}$ & 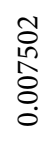 & $\begin{array}{l}\circ \\
: 0 \\
0 \\
0 \\
0 \\
0\end{array}$ & 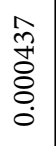 & 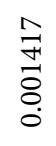 & 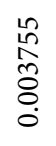 & 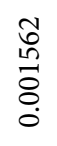 & 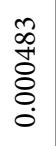 & $\begin{array}{l}\text { o } \\
\text { Oे } \\
8 \\
0 \\
\dot{0}\end{array}$ & 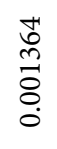 & \begin{tabular}{l}
$m$ \\
\multirow{J}{*}{} \\
$\stackrel{0}{0}$ \\
0 \\
$\dot{0}$
\end{tabular} & \multirow{2}{*}{$\begin{array}{l}\infty \\
0 \\
0 \\
0 \\
0 \\
0 \\
0\end{array}$} \\
\hline & 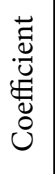 & $\begin{array}{l}\text { t. } \\
\stackrel{t}{+} \\
\stackrel{0}{0} \\
\stackrel{0}{0} \\
\stackrel{0}{0}\end{array}$ & 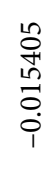 & $\begin{array}{l}\overrightarrow{\widetilde{p}} \\
\stackrel{0}{0} \\
0 \\
0\end{array}$ & 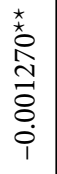 & 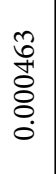 & 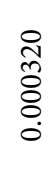 & 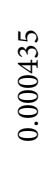 & 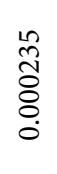 & $\begin{array}{l}0 \\
\text { ñ } \\
8 \\
0 \\
i \\
1\end{array}$ & 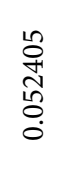 & 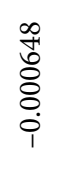 & 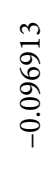 & \\
\hline \multirow{2}{*}{$\stackrel{12}{9}$} & 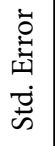 & \begin{tabular}{l}
$\stackrel{+}{0}$ \\
\multirow{7}{*}{} \\
$\stackrel{0}{0}$ \\
$\stackrel{0}{0}$
\end{tabular} & $\begin{array}{l}n \\
0 \\
h \\
0 \\
0 \\
0 \\
0\end{array}$ & 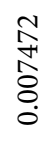 & $\begin{array}{l}\llcorner \\
\infty \\
\hat{0} \\
\dot{0} \\
0 \\
0\end{array}$ & $\begin{array}{l}\stackrel{+}{m} \\
\stackrel{+}{0} \\
\dot{0} \\
\dot{0}\end{array}$ & $\begin{array}{l}8 \\
\stackrel{+}{+} \\
8 \\
8 \\
0\end{array}$ & $\begin{array}{l}\overrightarrow{0} \\
\infty \\
\tilde{O} \\
\dot{0} \\
\dot{0}\end{array}$ & $\begin{array}{l}0 \\
\stackrel{0}{0} \\
8 \\
0 \\
0\end{array}$ & 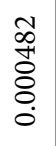 & $\begin{array}{l}\tilde{N} \\
\tilde{\delta} \\
\delta \\
0 \\
\dot{0}\end{array}$ & $\begin{array}{l}\infty \\
\stackrel{\infty}{0} \\
\stackrel{8}{8} \\
\dot{0}\end{array}$ & $\begin{array}{l}m \\
\infty \\
0 \\
\hat{0} \\
0 \\
0\end{array}$ & \multirow{2}{*}{$\begin{array}{l}\mathscr{L} \\
\infty \\
\infty \\
\stackrel{0}{0} \\
\infty \\
0 \\
0\end{array}$} \\
\hline & 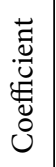 & 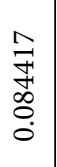 & $\begin{array}{l}\stackrel{2}{0} \\
\infty \\
\stackrel{0}{0} \\
0 \\
0\end{array}$ & $\begin{array}{l}\text { O } \\
\stackrel{1}{0} \\
0 \\
0 \\
1 \\
1\end{array}$ & $\begin{array}{l}n \\
2 \\
\circ \\
0 \\
0 \\
\dot{\varphi} \\
1\end{array}$ & $\begin{array}{l}m \\
\stackrel{m}{\$} \\
\delta \\
0 \\
0\end{array}$ & $\begin{array}{l}\text { त } \\
\widehat{\delta} \\
\delta \\
0 \\
0\end{array}$ & 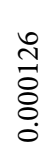 & 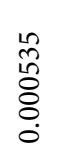 & 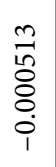 & $\begin{array}{l}\vec{J} \\
\vec{\sigma} \\
\dot{8} \\
\dot{0}\end{array}$ & 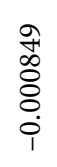 & 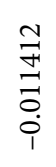 & \\
\hline \multirow{2}{*}{ 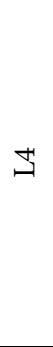 } & 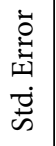 & 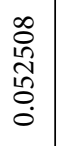 & $\begin{array}{l}\stackrel{n}{O} \\
\stackrel{+}{1} \\
0 \\
0 \\
0\end{array}$ & $\begin{array}{l}\text { ㄱ } \\
\text { Nิ } \\
\stackrel{8}{0} \\
0\end{array}$ & 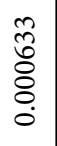 & 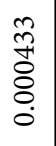 & 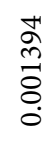 & 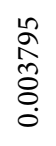 & $\begin{array}{l}\stackrel{2}{\infty} \\
\stackrel{0}{0} \\
\stackrel{0}{0} \\
\stackrel{0}{0}\end{array}$ & $\begin{array}{l}\text { ஓे } \\
\text { के } \\
8 \\
0 \\
0\end{array}$ & 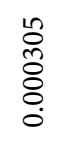 & $\begin{array}{l}\hat{\infty} \\
\infty \\
\stackrel{0}{0} \\
\stackrel{8}{0} \\
0\end{array}$ & $\begin{array}{l}\stackrel{n}{\vec{F}} \\
\stackrel{1}{\hat{O}} \\
\dot{0}\end{array}$ & \multirow{2}{*}{$\begin{array}{l}\tilde{O} \\
\widetilde{0} \\
\infty \\
0 \\
0\end{array}$} \\
\hline & 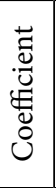 & 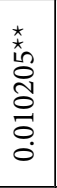 & $\begin{array}{l}2 \\
0 \\
0 \\
0 \\
0 \\
0 \\
1\end{array}$ & 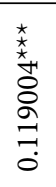 & 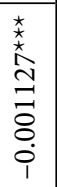 & $\begin{array}{l}\hat{y} \\
\text { ฟै } \\
0 \\
0 \\
0\end{array}$ & 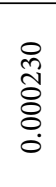 & $\begin{array}{l}\tilde{m} \\
\stackrel{8}{0} \\
\vdots \\
\dot{0}\end{array}$ & $\begin{array}{l}n \\
0 \\
0 \\
0 \\
0 \\
0\end{array}$ & 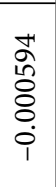 & $\begin{array}{l}n \\
8 \\
8 \\
0 \\
0 \\
0\end{array}$ & $\begin{array}{l}\stackrel{n}{\vec{J}} \\
\stackrel{\Xi}{0} \\
\stackrel{i}{i}\end{array}$ & $\begin{array}{l}\tilde{a} \\
\hat{0} \\
0 \\
0 \\
\dot{0}\end{array}$ & \\
\hline \multirow{2}{*}{$\stackrel{3}{9}$} & $\begin{array}{l}\dot{\overrightarrow{0}} \\
\dot{=} \\
\dot{y} \\
\dot{D}\end{array}$ & $\begin{array}{l}\hat{N} \\
\hat{N} \\
\stackrel{D}{0} \\
0\end{array}$ & $\begin{array}{l}\text { 오 } \\
\text { h } \\
\hat{n} \\
0 \\
0 \\
0\end{array}$ & 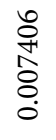 & $\begin{array}{l}0 \\
\ddot{0} \\
\vdots \\
0 \\
0\end{array}$ & $\begin{array}{l}\vec{H} \\
\stackrel{0}{0} \\
0 \\
0 \\
0\end{array}$ & 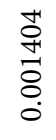 & 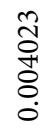 & $\begin{array}{l}\vec{H} \\
\stackrel{0}{\sigma} \\
\stackrel{0}{0}\end{array}$ & $\begin{array}{l}\text { શे } \\
\text { †े } \\
8 \\
0 \\
0\end{array}$ & $\begin{array}{l}n \\
0 \\
\tilde{o} \\
8 \\
0 \\
0\end{array}$ & $\begin{array}{l}\stackrel{n}{0} \\
m \\
\stackrel{8}{0} \\
\dot{0}\end{array}$ & $\begin{array}{l}m \\
0 \\
0 \\
0 \\
0 \\
0\end{array}$ & \multirow{2}{*}{$\begin{array}{l}\text { ⿵े } \\
\hat{0} \\
0 \\
0 \\
0\end{array}$} \\
\hline & 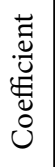 & 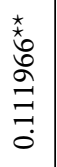 & $\begin{array}{l}n \\
0 \\
\infty \\
0 \\
0 \\
0 \\
0 \\
1\end{array}$ & $\begin{array}{l}\infty \\
\infty \\
\circ \\
\hat{\sigma} \\
\dot{0} \\
\dot{1}\end{array}$ & 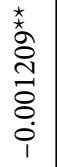 & 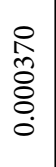 & $\begin{array}{l}8 \\
\text { م. } \\
8 \\
0 \\
0\end{array}$ & $\begin{array}{l}\text { ले } \\
\bar{\Xi} \\
\dot{0} \\
\dot{1}\end{array}$ & 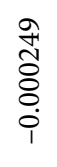 & $\begin{array}{l}\vec{\infty} \\
0 \\
0 \\
0 \\
0 \\
i\end{array}$ & $\begin{array}{l}\text { no } \\
\hat{N} \\
\vdots \\
0 \\
0\end{array}$ & $\begin{array}{l}\overrightarrow{0} \\
0 \\
8 \\
0 \\
0 \\
i\end{array}$ & 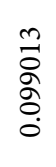 & \\
\hline \multirow{2}{*}{$\mathcal{I}$} & 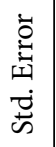 & $\begin{array}{l}n \\
0 \\
\infty \\
\tilde{0} \\
\hat{0} \\
0 \\
0\end{array}$ & 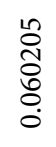 & 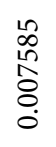 & 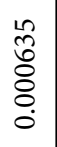 & 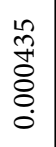 & 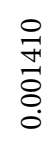 & 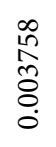 & 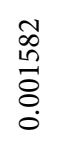 & 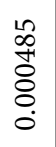 & $\begin{array}{l}8 \\
8 \\
\delta \\
\delta \\
0 \\
\dot{0}\end{array}$ & $\begin{array}{l}\stackrel{n}{\tilde{F}} \\
\stackrel{+}{8} \\
\dot{0}\end{array}$ & $\begin{array}{l}\frac{n}{0} \\
\stackrel{1}{\hat{0}} \\
0 \\
0\end{array}$ & \multirow{2}{*}{ 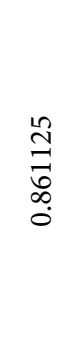 } \\
\hline & 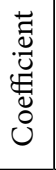 & 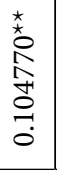 & $\begin{array}{l}n \\
0 \\
0 \\
0 \\
0 \\
0 \\
1\end{array}$ & 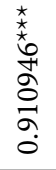 & 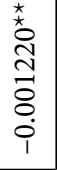 & 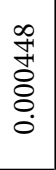 & $\begin{array}{l}\stackrel{0}{\widehat{N}} \\
\stackrel{0}{0} \\
\stackrel{0}{0}\end{array}$ & 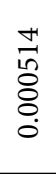 & 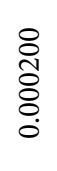 & $\begin{array}{l}\overrightarrow{\tilde{n}} \\
\hat{2} \\
0 \\
0 \\
\dot{0}\end{array}$ & 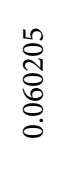 & $\begin{array}{l}0 \\
0 \\
0 \\
0 \\
0 \\
0 \\
1\end{array}$ & $\begin{array}{l}\stackrel{\Xi}{\sigma} \\
0 \\
0 \\
0 \\
i\end{array}$ & \\
\hline \multirow{2}{*}{$\exists$} & 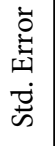 & $\begin{array}{l}\text { वे } \\
\stackrel{0}{0} \\
0 \\
0 \\
0 \\
0\end{array}$ & $\begin{array}{l}n \\
0 \\
0 \\
0 \\
0 \\
0 \\
0\end{array}$ & $\begin{array}{l}\infty \\
\stackrel{\circ}{1} \\
\hat{\circ} \\
\stackrel{0}{0} \\
0\end{array}$ & $\begin{array}{l}\overrightarrow{\hat{\sigma}} \\
\dot{8} \\
\dot{0} \\
\dot{0}\end{array}$ & \begin{tabular}{l}
$\stackrel{0}{+}$ \\
\multirow{4}{0}{} \\
8 \\
0 \\
0
\end{tabular} & $\begin{array}{l}\text { ô } \\
\stackrel{+}{1} \\
\dot{8} \\
\dot{0}\end{array}$ & $\begin{array}{l}\frac{0}{1} \\
\text { Oे } \\
0 \\
0\end{array}$ & 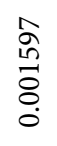 & $\begin{array}{l}\vec{\infty} \\
\stackrel{\infty}{0} \\
8 \\
0 \\
0\end{array}$ & $\begin{array}{l}\stackrel{1}{0} \\
\tilde{\delta} \\
\dot{\delta} \\
\dot{0}\end{array}$ & $\begin{array}{l}8 \\
\stackrel{0}{0} \\
0 \\
8 \\
0\end{array}$ & $\begin{array}{l}m \\
0 \\
0 \\
0 \\
0 \\
0\end{array}$ & \multirow{2}{*}{ 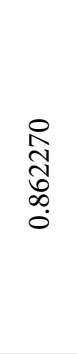 } \\
\hline & 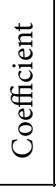 & 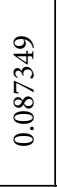 & 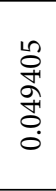 & 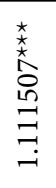 & 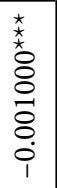 & 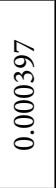 & 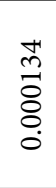 & 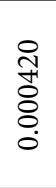 & 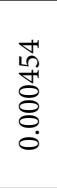 & $\begin{array}{l}n \\
\tilde{n} \\
0 \\
0 \\
0 \\
i\end{array}$ & 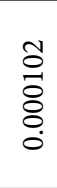 & $\begin{array}{l}\infty \\
\infty \\
0 \\
0 \\
0 \\
0 \\
i\end{array}$ & $\begin{array}{l}\tilde{N} \\
\infty \\
\Xi \\
0 \\
\dot{0} \\
1\end{array}$ & \\
\hline & 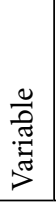 & $u$ & 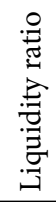 & 岕 & 岕 & 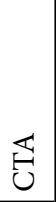 & 虹 & 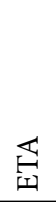 & 耍 & 㞱 & 今心 & $\stackrel{U}{U_{I}}$ & $\overrightarrow{\mid \vec{I}}$ & 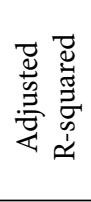 \\
\hline
\end{tabular}




\begin{tabular}{|c|c|c|c|c|c|c|c|c|c|c|c|c|c|c|}
\hline & 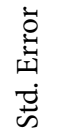 & 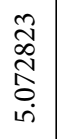 & 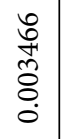 & $\begin{array}{l}m \\
\stackrel{n}{\sharp} \\
\tilde{D} \\
0 \\
0\end{array}$ & $\begin{array}{l}\vec{F} \\
0 \\
\stackrel{0}{0} \\
0 \\
0\end{array}$ & $\begin{array}{l}\stackrel{2}{2} \\
\alpha \\
o \\
\stackrel{+}{0} \\
0\end{array}$ & 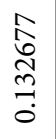 & $\begin{array}{c}0 \\
\delta \\
\sigma \\
\tilde{n} \\
\tilde{0} \\
0\end{array}$ & 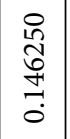 & 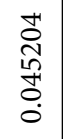 & $\begin{array}{l}\hat{0} \\
\stackrel{0}{0} \\
\approx \\
0 \\
0\end{array}$ & 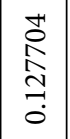 & $\begin{array}{l}\vec{F} \\
\stackrel{\tilde{H}}{0} \\
\dot{0}\end{array}$ & \multirow{2}{*}{ 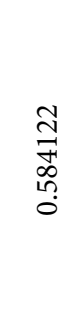 } \\
\hline & 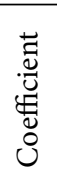 & \begin{tabular}{l}
$\stackrel{2}{\alpha}$ \\
2 \\
\multirow{7}{*}{} \\
$i \mathfrak{1}$
\end{tabular} & $\begin{array}{l}\stackrel{\star}{\star} \\
\stackrel{\mathbb{I}}{\mathbf{I}} \\
\stackrel{\hat{0}}{0} \\
\dot{0}\end{array}$ & 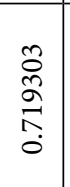 & 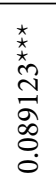 & $\begin{array}{l}\vec{\partial} \\
\vec{H} \\
\hat{0} \\
\dot{0}\end{array}$ & 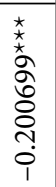 & $\begin{array}{l}m \\
\vec{n} \\
0 \\
0 \\
0 \\
i \\
1\end{array}$ & 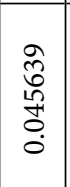 & $\begin{array}{l}\stackrel{\vartheta}{\mathrm{U}} \\
\infty \\
\tilde{O} \\
0 \\
0\end{array}$ & $\begin{array}{l}0 \\
\stackrel{0}{0} \\
\infty \\
0 \\
0 \\
0 \\
1\end{array}$ & $\mid \begin{array}{c}\not 1 \\
0 \\
0 \\
0 \\
0 \\
0 \\
0 \\
1\end{array}$ & 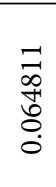 & \\
\hline \multirow{2}{*}{13} & 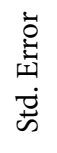 & 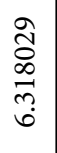 & $\begin{array}{l}\overrightarrow{0} \\
0 \\
\widetilde{0} \\
\dot{0} \\
\dot{0}\end{array}$ & $\begin{array}{l}\stackrel{2}{2} \\
\stackrel{\tilde{F}}{\tilde{H}} \\
\hat{0}\end{array}$ & 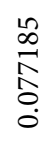 & 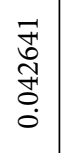 & 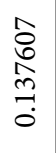 & $\begin{array}{c}m \\
n \\
\tilde{n} \\
\tilde{n} \\
0 \\
0\end{array}$ & 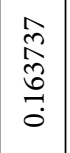 & $\begin{array}{l}\text { 今े } \\
\text { N } \\
\text { ثे } \\
0\end{array}$ & $\begin{array}{l}\stackrel{0}{0} \\
\stackrel{0}{0} \\
\ddot{0} \\
0 \\
0\end{array}$ & 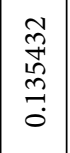 & $\begin{array}{l}\vec{F} \\
\vec{F} \\
\stackrel{0}{0} \\
0 \\
0\end{array}$ & \multirow{2}{*}{ 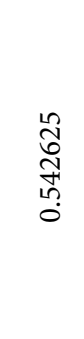 } \\
\hline & 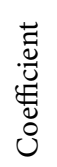 & $\begin{array}{l}0 \\
\stackrel{0}{n} \\
\hat{\alpha} \\
\tilde{\sigma} \\
0\end{array}$ & 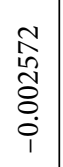 & $\begin{array}{l}\infty \\
\infty \\
10 \\
10 \\
0 \\
0 \\
0\end{array}$ & 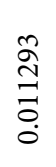 & $\begin{array}{l}\infty \\
\vdots \\
\vdots \\
\vdots \\
\vdots \\
0\end{array}$ & $\begin{array}{l}8 \\
0 \\
0 \\
0 \\
0 \\
0 \\
1\end{array}$ & $\begin{array}{l}+ \\
8 \\
0 \\
0 \\
1 \\
1 \\
1\end{array}$ & $\left|\begin{array}{c}0 \\
0 \\
0 \\
n \\
0 \\
0 \\
1\end{array}\right|$ & $\begin{array}{l}\hat{O} \\
\text { Iิ } \\
\text { Oे }\end{array}$ & 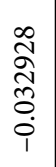 & 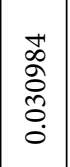 & $\begin{array}{l}\stackrel{0}{\vec{m}} \\
\stackrel{0}{\overrightarrow{0}} \\
\dot{0}\end{array}$ & \\
\hline \multirow{2}{*}{$\exists$} & $\begin{array}{l}\ddot{0} \\
\text { ज्ञ } \\
\overrightarrow{\dot{D}}\end{array}$ & $\begin{array}{l}\vec{n} \\
\widetilde{\widetilde{Z}} \\
\text { त̂ } \\
\text { in }\end{array}$ & $\begin{array}{l}\stackrel{2}{a} \\
\stackrel{0}{0} \\
\vdots \\
0\end{array}$ & $\begin{array}{l}a \\
\hat{0} \\
\stackrel{0}{0} \\
\stackrel{0}{0}\end{array}$ & $\begin{array}{l}\text { F } \\
\text { ते } \\
\stackrel{0}{0}\end{array}$ & 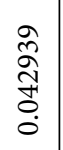 & $\begin{array}{l}\hat{b} \\
\infty \\
m \\
0 \\
0\end{array}$ & $\begin{array}{c}\tilde{m} \\
\tilde{\delta} \\
\tilde{0} \\
\tilde{0}\end{array}$ & 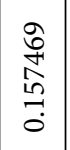 & 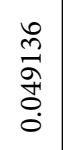 & $\begin{array}{l}\overrightarrow{\widetilde{\Xi}} \\
\text { Oे } \\
0 \\
0\end{array}$ & 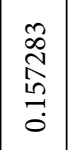 & $\begin{array}{l}\overrightarrow{1} \\
0 \\
0 \\
0 \\
0\end{array}$ & \multirow{2}{*}{ 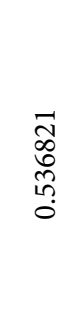 } \\
\hline & 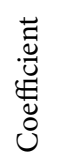 & $\begin{array}{l}\overrightarrow{\hat{O}} \\
\hat{0} \\
\hat{2} \\
\stackrel{\oplus}{1} \\
\end{array}$ & 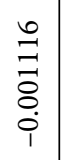 & 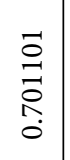 & $\begin{array}{l}\stackrel{\Delta}{d} \\
\infty \\
0 \\
0 \\
0\end{array}$ & $\begin{array}{l}\hat{2} \\
\hat{2} \\
0 \\
0 \\
0\end{array}$ & \begin{tabular}{c}
$\vec{\infty}$ \\
\multirow{4}{*}{} \\
$\stackrel{4}{0}$ \\
$\overrightarrow{0}$ \\
1
\end{tabular} & $\begin{array}{c}\hat{\Omega} \\
\hat{n} \\
\infty \\
0 \\
0 \\
1 \\
1\end{array}$ & $\left|\begin{array}{l}8 \\
\vdots \\
\vdots \\
1 \\
0\end{array}\right|$ & 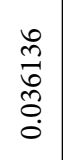 & 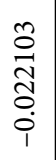 & $\begin{array}{l}n \\
2 \\
\hat{\sigma} \\
\vdots \\
0 \\
0 \\
1\end{array}$ & $\begin{array}{l}\bar{n} \\
\hat{n} \\
\infty \\
0 \\
0\end{array}$ & \\
\hline \multirow{2}{*}{3} & 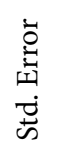 & $\begin{array}{l}\hat{2} \\
\hat{2} \\
\hat{0} \\
\infty \\
+ \\
+\end{array}$ & $\begin{array}{l}\stackrel{\circ}{7} \\
\stackrel{7}{0} \\
\stackrel{0}{0} \\
\stackrel{0}{0}\end{array}$ & $\begin{array}{l}\widetilde{d} \\
\tilde{N} \\
\tilde{0} \\
0 \\
0\end{array}$ & $\begin{array}{l}\text { Oे } \\
0 \\
0 \\
0 \\
0 \\
0\end{array}$ & 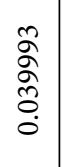 & 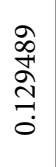 & 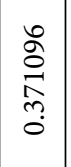 & $\left|\begin{array}{c}m \\
+ \\
\infty \\
\infty \\
+1 \\
0 \\
0\end{array}\right|$ & \begin{tabular}{l}
$\vec{H}$ \\
$\stackrel{H}{*}$ \\
\multirow{+}{0}{} \\
0
\end{tabular} & $\begin{array}{l}\stackrel{n}{a} \\
\overrightarrow{0} \\
\tilde{\sigma} \\
0 \\
0\end{array}$ & $\mid \begin{array}{c}\tilde{0} \\
\widetilde{N} \\
\stackrel{-1}{0} \\
0\end{array}$ & $\begin{array}{l}\Xi \\
\bar{\Xi} \\
0 \\
0 \\
0\end{array}$ & \multirow{2}{*}{$\begin{array}{l}\stackrel{0}{N} \\
\hat{\Xi} \\
\stackrel{0}{0} \\
0\end{array}$} \\
\hline & 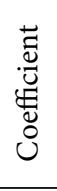 & 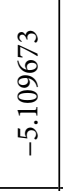 & 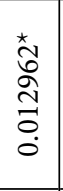 & 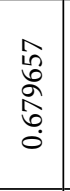 & $\begin{array}{l}\text { on } \\
\hat{\alpha} \\
\hat{0} \\
0 \\
0\end{array}$ & $\begin{array}{l}\tilde{O} \\
\hat{H} \\
\tilde{N} \\
0 \\
0 \\
0\end{array}$ & 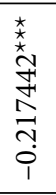 & 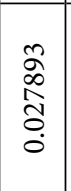 & $\begin{array}{l}\overrightarrow{0} \\
0 \\
\stackrel{0}{+} \\
\stackrel{0}{0}\end{array}$ & $\begin{array}{l}\stackrel{2}{2} \\
\hat{\imath} \\
\hat{0} \\
0\end{array}$ & 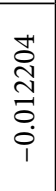 & 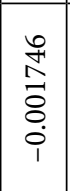 & $\begin{array}{l}\vec{F} \\
\text { +5 } \\
0 \\
0 \\
0 \\
0\end{array}$ & \\
\hline \multirow{2}{*}{$\mathcal{I}$} & 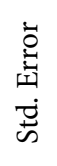 & 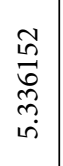 & $\begin{array}{l}0 \\
0 \\
0 \\
o ̛ ⿱ 宀 \\
0 \\
0\end{array}$ & 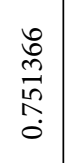 & 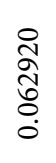 & $\begin{array}{l}\stackrel{0}{\overrightarrow{3}} \\
\stackrel{3}{0} \\
\stackrel{0}{0}\end{array}$ & $\begin{array}{l}\hat{0} \\
\infty \\
心 \\
\stackrel{0}{0} \\
\end{array}$ & $\begin{array}{c}\overrightarrow{0} \\
\tilde{N} \\
\hat{n} \\
0\end{array}$ & $\left|\begin{array}{c}0 \\
0 \\
0 \\
0 \\
10 \\
0 \\
0\end{array}\right|$ & 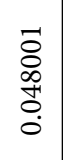 & $\begin{array}{l}\text { Lे } \\
0 \\
0 \\
0 \\
0 \\
0\end{array}$ & $\mid \begin{array}{c}\vec{\infty} \\
\underset{I}{I} \\
\overrightarrow{0}\end{array}$ & $\begin{array}{l}\overrightarrow{0} \\
0 \\
0 \\
0 \\
0\end{array}$ & \multirow{2}{*}{ 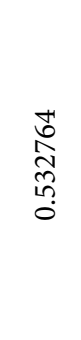 } \\
\hline & 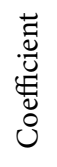 & 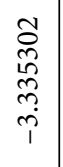 & 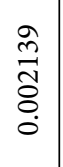 & 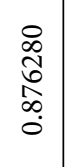 & $\begin{array}{l}\vec{W} \\
\infty \\
0 \\
0 \\
0 \\
0\end{array}$ & $\begin{array}{l}\tilde{O} \\
0 \\
0 \\
0 \\
0\end{array}$ & $\begin{array}{l}\underset{m}{H} \\
\tilde{n} \\
\stackrel{n}{0} \\
i\end{array}$ & 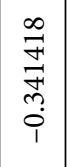 & 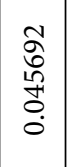 & 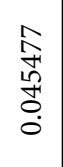 & 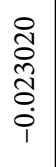 & 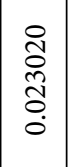 & $\begin{array}{l}\bar{\sigma} \\
\text { Oे } \\
0 \\
0\end{array}$ & \\
\hline & 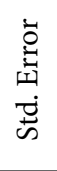 & \begin{tabular}{l}
0 \\
\multirow{0}{0}{} \\
0 \\
$i n$ \\
$i n$ \\
$i n$
\end{tabular} & $\begin{array}{l}\widetilde{\widetilde{N}} \\
\infty \\
0 \\
0 \\
0\end{array}$ & 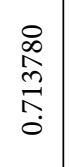 & 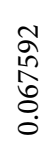 & $\begin{array}{l}\mathcal{I} \\
\infty \\
\mathbb{7} \\
0 \\
0\end{array}$ & $\begin{array}{l}\vec{n} \\
\tilde{n} \\
\stackrel{0}{0} \\
0\end{array}$ & $\begin{array}{l}0 \\
\tilde{m} \\
\tilde{0} \\
\tilde{n} \\
0 \\
0\end{array}$ & $\left|\begin{array}{c}n \\
\infty \\
\infty \\
n \\
0 \\
0\end{array}\right|$ & $\begin{array}{l}0 \\
\hat{n} \\
\vdots \\
0 \\
0 \\
0\end{array}$ & 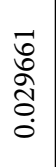 & 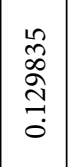 & 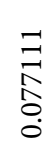 & \multirow{2}{*}{$\begin{array}{l}0 \\
\infty \\
0 \\
\tilde{N} \\
\hat{n} \\
0\end{array}$} \\
\hline & 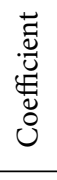 & $\begin{array}{l}2 \\
\stackrel{2}{0} \\
0 \\
\stackrel{9}{+} \\
\stackrel{1}{i}\end{array}$ & 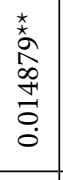 & 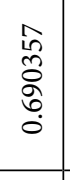 & 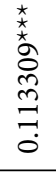 & $\begin{array}{l}0 \\
\stackrel{0}{10} \\
\tilde{O} \\
0 \\
0\end{array}$ & $\begin{array}{l}\vec{n} \\
2 \\
\infty \\
\overrightarrow{0} \\
1\end{array}$ & $\begin{array}{c}\infty \\
\infty \\
\mathbb{1} \\
0 \\
0 \\
0 \\
1\end{array}$ & $\mid \begin{array}{l}\hat{A} \\
\Delta \\
0 \\
0 \\
0\end{array}$ & 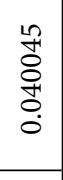 & $\begin{array}{l}\hat{N} \\
\stackrel{ }{0} \\
0 \\
\dot{1} \\
1\end{array}$ & $\begin{array}{c}+ \\
0 \\
0 \\
0 \\
\tilde{O} \\
0 \\
1\end{array}$ & $\begin{array}{l}\vec{\sigma} \\
\stackrel{\sigma}{\sigma} \\
0 \\
0\end{array}$ & \\
\hline & 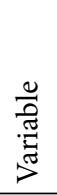 & 0 & 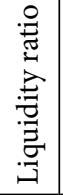 & 災 & 岕 & $\overleftrightarrow{U}$ & 空 & 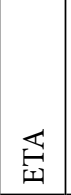 & 㟐 & 㞱 & 今િ & $\overrightarrow{\vec{\theta}}$ & $\stackrel{\text { II }}{\mathrm{G}}$ & 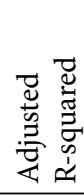 \\
\hline
\end{tabular}




\section{Conclusions}

The financial crisis of 2007 revealed the importance of establishing a level of liquidity sufficient to cope with adverse conditions and have highlighted serious flaws in the methods of management of liquidity risk of individual banks.

The purpose of this paper is to analyze the relationship between liquidity risk and financial performance of Moroccan banks and to define the determinants of bank's performance in Morocco. To do this, we first evaluate Moroccan bank's liquidity positions and bank's financial performance through different liquidity ratios. We then identify determinants of Moroccan bank's performance using a panel data regression and analyze the relationship between liquidity risk and financial performance of Moroccan banks.

We conclude that Moroccan bank's performance is mainly determined by 7 determinants: liquidity ratio, size of banks, logarithm of the total assets squared, external funding to total liabilities, share of own bank's capital of the bank's total assets, foreign direct investments, unemployment rate and the realization of the financial crisis variable. Banks' performance depends positively on size of banks, on foreign direct investments and on the realization of the financial crisis and negatively on external funding to total liabilities, on share of own bank's capital of the bank's total assets and on unemployment rate while the dependence between bank performance and liquidity ratios and bank performance and logarithm of the total assets squared depend on the model used.

Results show that we cannot say that liquid banks are more efficient than illiquid banks. Large banks and banks with a low share of own bank's capital of the bank's total assets are more efficient while banks depending on external funding are less efficient. Bank performance decreases with the financial crisis and increases when the foreign direct investments grow. Bank's performance increase when unemployment rate decreases. However, the correlation of logarithm of the total assets squared and bank's performance depends on liquidity ratio used, while equity to total assets and growth rate of gross domestic product have no impact on bank's performance.

\section{References}

Ariffin, N. M. 2012. Liquidity risk management and financial performance in Malaysia: empirical evidence from Islamic banks, Aceh International Journal of Social Sciences 1(2): 68-75.

Athanasoglou, P. P.; Delis, M. D.; Staikouras, C. K. 2006. Determinants of bank profitability in the South Eastern European Region, Journal of Financial Decision Making 2(47):1-17.

Attijariwafa Bank. 2013. Annual reports ATTIJARIWAFA BANK 2001-2012 [online], [cited 2 January 2013]. Available from Internet: http://www.attijariwafabank.com/ESPACEFINANCIER/Pages/Rapportsannuels.aspx
Banque Centrale Populaire. 2013. Annual reports BANQUE CENTRALE POPULAIRE 2001-2012 [online], [cited 2 January 2013]. Available from Internet: http://www.gbp.ma/InformationFinanciere/Pages/CommunicationFinanciere.aspx

Barth, J. R.; Nolle, D. E.; Phumiwasana, T.; Yago, G. 2003. A crosscountry analysis of the bank supervisory framework and bank performance, Financial Markets, Institutions and Instruments 12: 67-120. http://dx.doi.org/10.1111/1468-0416.t01-2-00001

Basel Committee on Banking Supervision. 2000. Sound practices for managing liquidity in banking organizations. Bank for International Settlement. $21 \mathrm{p}$.

Basel Committee on Banking Supervision, 2008. Principles for Sound Liquidity Risk Management and Supervision. Bank for International Settlements. 44p.

Basel Committee on Banking Supervision. 2010. Basel III: International framework for liquidity risk measurement, standards and monitoring. Bank for International Settlement. $47 \mathrm{p}$.

BMCE Bank. 2013. Annual reports BANQUE MAROCAINE DU COMMERCE EXTERIEUR 2001-2012 [online], [cited 2 January 2013]. Available from Internet: http://www.bmcebank. $\mathrm{ma} /$ front.aspx?sectionId $=60$

BMCI Groupe BNP Paribas. 2013. Annual reports BANQUE MAROCAINE POUR LE COMMERCE ET L'INDUSTRIE 2001-2012 [online], [cited 2 January 2013]. Available from Internet: http://www.bmci.ma/fr/pid3660/rapports-annuelsdocs-financiers.html

Bourke, P. 1989. Concentration and other determinants of bank profitability in Europe, North America and Australia, Journal of Banking and Finance 13: 65-79. http://dx.doi.org/10.1016/0378-4266(89)90020-4

Brunnermeier, M. K. 2009. Deciphering the liquidity and credit crunch 2007-2008, Journal of Economic Perspectives 23(1): 77-100. http://dx.doi.org/10.1257/jep.23.1.77

CIH Bank. 2013. Annual reports CREDIT IMMOBILIER ET HOTELIER 2001-2012 [online], [cited 2 January 2013]. Available from Internet: http://www.cihbank.ma/corporate/ espace-financier/rapport-activites

Chen, H.-J.; Kuo, C.-J.; Shen, C.-H. 2001. Determinants of net interest margins in Taiwan banking industry, Journal of Financial Studies 9: 47-83.

Chen, Y.-K.; Kao, L.-F.; Shen, C.-H.; Yeh, C.-Y. 2009. Bank liquidity risk and performance, in Proc. of the Asian Finance Association International Conference 2010, 30 June, 2010, Hong Kong.

CREDIT DU MAROC. 2013. Annual reports CREDIT DU MAROC 2001-2012 [online], [cited 2 January 2013]. Available from Internet: http://www.cdm.co.ma/www/fr/website/ rapport-annuels

Demirgüç-Kunt, A.; Huizinga, H. 1999. Determinants of commercial bank interest margins and profitability: some international evidence, World Bank Economic Review 13: 379-408. http://dx.doi.org/10.1093/wber/13.2.379

Demirgüç-Kunt, A.; Laeven, L.; Levine, R. 2003. The impact of bank regulations, concentration, and institutions on bank margins, World Bank Policy Research Working Paper $3030.60 \mathrm{p}$.

Diamond, D. W.; Dybvig, P. H. 1983. Bank runs, deposit insurance, and liquidity, Journal of Political Economy 91(3): 401-419. http://dx.doi.org/10.1086/261155 
Dickey, D. A.; Fuller, W. A. 1979. Distribution of the estimators for autoregressive time series with a unit root, Journal of the American Statistical Association 74: 427-431.

European Central Bank. 2010. Beyond ROE - how to measure bank performance. The Report On EU Banking Structures. 43 p.

Fielding, D.; Shortland, A. 2005. Political violence and excess liquidity in Egypt, Journal of Development Studies 41: 542557. http://dx.doi.org/10.1080/00220380500092580

Geršl, A.; Komárková, Z. 2009. Liquidity risk and banks' bidding behavior: evidence from the global financial crisis, Czech Journal of Economics and Finance 59(6): 577-592.

Groupe Crédit Agricole du Maroc. 2013. Annual reports CREDIT AGRICOLE 2001-2012 [online], [cited 2 January 2013]. Available from Internet: http://www.creditagricole.ma/ EspaceMedia/Publication/Pages/Archive.aspx?Cat=UmF wcG9ydCtkJ2FjdGl2aXQlYzMlYTlz

Hemple, G. H.; Simonson, D. G. 2008. Bank management: text and cases. 6th ed. John Wiley And Sons. 704 p.

International Monetary Fund. 2013. Databases of the International Monetary Fund 2001-2012 [online], [cited 10 January 2013]. Available from Internet: http://www.imf.org/external/data. htm

Kosmidou, K.; Tanna, S.; Pasiouras, F. 2005, Determinants of profitability of domestic UK commercial banks: panel evidence from the period 1995-2002, in Money Macro and Finance (MMF) Research Group Conference, 1 June 2005, Rethymno, Greece. 20 p.

Kosmidou, K. 2008. The determinants of banks' profits in Greece during the period of EU financial integration, Managerial Finance 34: 146-159. http://dx.doi.org/10.1108/03074350810848036

Lucchetta, M. 2007. What do data say about monetary policy, bank liquidity and bank risk taking?, Economic Notes by Banca Monte dei Paschi di Siena SpA 36(2):189-203.

Matz, L.; Neu, P. 2006. Liquidity risk measurement and management: a practitioner's guide to global best practices. John Wiley and Sons. 350 p. http://dx.doi.org/10.1002/9781118390399

Molyneux, P.; Thornton, J. 1992. Determinants of European bank profitability: a note, Journal of Banking and Finance 16: 1173-1178. http://dx.doi.org/10.1016/0378-4266(92)90065-8
Moore, W. 2010. How do financial crises affect commercial bank liquidity? Evidence from Latin America and the Caribbean. MPRA Paper. 24 p.

Moro, B. 2013. Lessons from the European economic and financial great crisis: a survey, European Journal of Political Economy (Corrected Proof) [online], [cited 3 July 2013]. Available from Internet: http://www.siecon.org/online/wp-content/ uploads/2013/09/Moro.pdf

Moroccan High Commission for Planning. 2013. Databases of the Moroccan High Commission for Planning 2001-2012 [online], [cited 11 January 2013]. Available from Internet: http://www. hcp.ma/Chomage_r70.html

Naceur, S. B.; Kandil, M. 2009. The impact of capital requirements on banks' cost of intermediation and performance: the case of Egypt, Journal of Economics and Business 61: 70-89. http://dx.doi.org/10.1016/j.jeconbus.2007.12.001

Pasiouras, F.; Kosmidou, K. 2007. Factors influencing the profitability of domestic and foreign commercial banks in the European Union, Research in International Business and Finance 21: 222-237. http://dx.doi.org/10.1016/j.ribaf.2006.03.007

Poorman, F. Jr.; Blake, J. 2005. Measuring and modeling liquidity risk: new ideas and metrics, Financial Managers Society Inc. White Paper 2005.

Saunders, A.; Cornett, M. M. 2006. Financial institutions management: a risk management approach. McGraw-Hill. 884 p.

Societe Generale. 2013. Annual reports SOCIETE GENERALE MAROCAINE DE BANQUES 2001-2012 [online], [cited 2 January 2013]. Available from Internet: http://www.sgmaroc. com/index.php?id=4

Vento, A. G.; La Ganga, P. 2009. Bank liquidity Risk management and supervision: which lessons from recent market turmoil?, Journal of Money, Investment and Banking 10: 79-126.

World Bank. 2013. Databases of the World Bank 2001-2012 [online], [cited 10 January 2013]. Available from Internet: http:// data.worldbank.org/

Yeager, F. C.; Seitz, N. E. 1989. Financial institution management: text and cases. $3^{\text {rd }}$ ed. Englewood Cliffs: Prentice Hall Inc. 496 p.

El Mehdi FERROUHI. PhD researcher in finance. Faculty of Law and Economics, Mohammed V Agdal University, Rabat, Morocco. Research interests: Liquidity risk, financial risks management, financial crisis, market microstructure. 